\title{
Redundant and Self-Healing Drive System for Pure EV Based on Low Voltage Building Blocks
}

\author{
Giuseppe Guidi*, Tore M. Undeland*
}

\begin{abstract}
An ideal interface between the energy tank on board of an EV and the electric motor used for propulsion should have a number of desirable characteristics: it should be efficient, reliable, safe, cheap, light and small, just to mention a few. Priority and relevance of each item may vary widely with the particular application. In this paper, the cascaded multilevel topology is proposed, mainly for those applications where reliability and availability is a major concern. It is shown that the proposed topology is inherently redundant, and that a self-healing system can be built around the cascaded converter with relatively little effort. Theoretical and practical aspects like voltage generation and optimal energy extraction within the system are discussed, and main concepts are proven by experimental results.
\end{abstract}

Keywords: Battery Management System (BMS), Charge Equalization, Power Electronics, Converter, Reliability.

\section{INTRODUCTION}

The use of multilevel cascaded inverter as power electronics interface between the battery pack on board of pure electric vehicles and the three-phase electric motor has been proposed in [1]. Obvious advantage of this multilevel topology, when compared to conventional 2-level inverter, is the reduction of the operating voltage across each switch. It is therefore possible to achieve operation at very high voltage, without explicit series connection of elementary switches. However, in automotive there is no need for very high voltage and the aim of the multilevel connection is to break up the whole DC link voltage, usually in the order of few hundred volts, in small steps that allow for use of extremely efficient low voltage switching devices (MOSFETs). Breaking up the DC link voltage leads also to other very desirable features, like the reduction of harmonic contents at the inverter output, the possible reduction of electromagnetic interference (EMI), increased safety and accessibility of the system due to the absence of high voltage when the inverter is not being operated. In addition to the above mentioned aspects that were already discussed in [1], there is at least one more topological property of cascaded inverters that deserves close attention: inherent redundancy. In the proposed architecture, failure of one (or more than one) block does not cause the whole system to shut down. In fact, the system, if properly engineered, is able to reconfigure itself

\footnotetext{
* Norwegian University of Science and Technology, NTNU

Trondheim, Norway

giuseppe.guidi@elkraft.ntnu.no

Tore.Undeland@elkraft.ntnu.no
}

(self-healing) with no need for human intervention and to resume operation almost as usual, with only a slight derating in maximum output power capabilities and virtually no loss in driving range. Since the DC-link is physically split into several isolated banks, charge and discharge of each bank can be independently controlled, allowing for ideal load sharing between all banks.

\section{ARCHITECTURE DESCRIPTION}

The basic H-Bridge multilevel converter topology is depicted in Fig.1. Any number $\mathrm{N}$ of independent voltage sources (building blocks) consisting of a flying DC source and an H-Bridge are series connected, in order to elevate the output voltage capabilities to plus/minus the sum of all DC voltage sources forming the series connection:

$$
-\sum_{i=1}^{N} V_{D C, i} \leq V_{\text {out }}(t) \leq \sum_{i=1}^{N} V_{D C, i}
$$

In its basic form, the multilevel cascaded converter is a single phase voltage source. However, due to the flying nature of each local DC source, a three phase topology can be obtained simply by Wye (or Delta) connecting three single phase blocks, as shown in Fig.2.

The flying DC source of every building block may simply consist of a battery bank; according to the battery technology and the vehicle requirements, an ultracapacitor (UC) bank can also be included in the building block, as shown in Fig.1. The idea behind a battery-ultracapacitor hybrid energy source is to use the power dense ultracapacitors to supply the short-term peak power to the load, relieving stress on the energy dense battery thereby improving driving range and battery lifetime. Effectiveness of battery-UC 


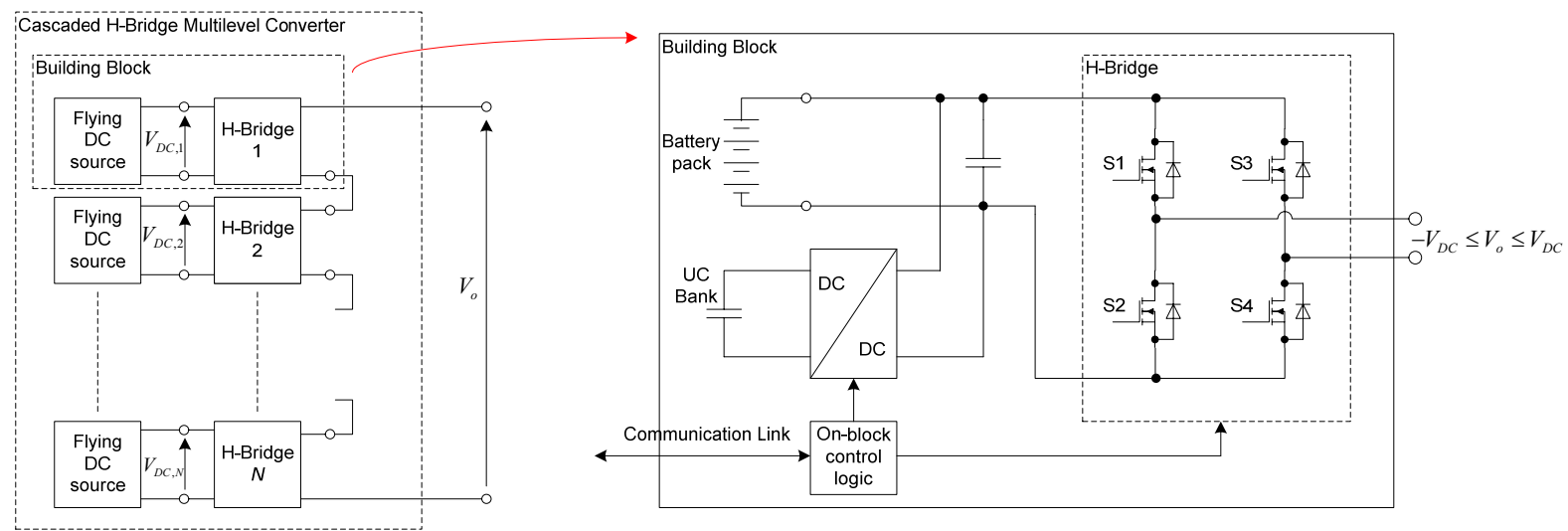

Fig. 1 - Basic configuration of Cascaded H-Bridge Multilevel converter, and related building block

combination in EVs has been subject of extensive study $[2,3]$, and is beyond the scope of this paper. In fact, the nature of the flying DC source has no influence on the redundancy and self-healing properties of the system.

\subsection{Operating Principle}

Due to the well known H-Bridge topology, at any given instant each building block is able to generate at its output terminals three different voltage levels:

$$
\begin{cases}+V_{D C} & \text { if } \mathrm{S} 1 \text { and } \mathrm{S} 4 \text { are } \mathrm{ON} ; \\ -V_{D C} & \text { if } \mathrm{S} 2 \text { and } \mathrm{S} 3 \text { are } \mathrm{ON} \\ 0 & \text { if } \mathrm{S} 1 \text { and } \mathrm{S} 3 \text { are } \mathrm{ON}, \text { or } \mathrm{S} 2 \text { and } \mathrm{S} 4 \text { are } \mathrm{ON} ;\end{cases}
$$

In the very common case of identical DC voltage sources for all building blocks, the resulting output voltage of the converter in Fig. 1 is then discretized with $(2 N+1)$ levels, the output voltage step being equal to the individual DC voltage $V_{D C}$. Any PWM technique can then be used, if necessary, to generate the desired average output voltage over a given switching period $T_{s w}$. The easiest technique would be to operate all but

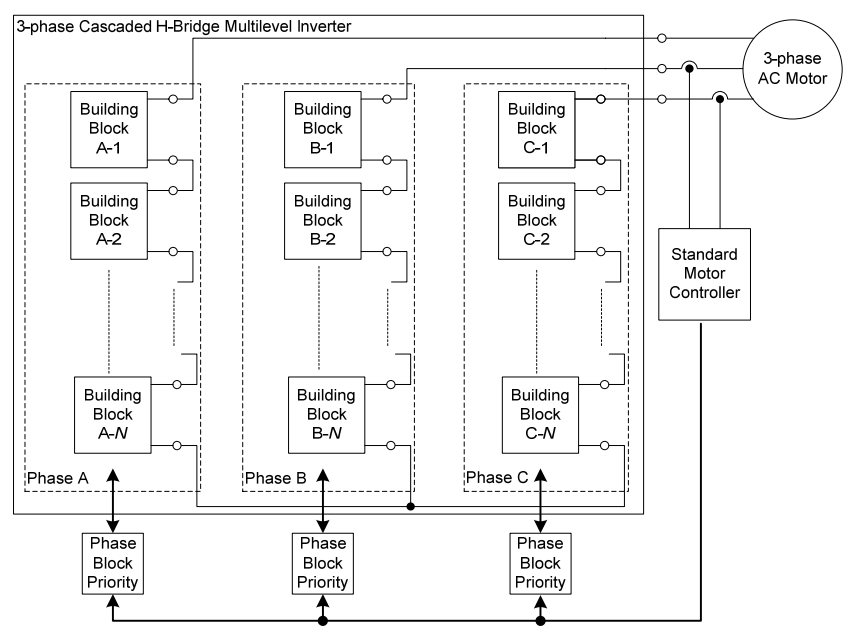

Fig. 2 - Three phase drive system based on cascaded H-Bridge multilevel topology one building block in continuous mode over a switching period, in order to minimize switching losses.

An important characteristic of the cascaded topology is that all individual blocks in a given phase chain are indistinguishable, as far as output voltage generation is concerned, provided they all have the same DC voltage behind the H-Bridge. This property can be exploited in order to achieve load sharing among the $N$ blocks, as described in section 2.3.

\subsection{Output Voltage Generation}

Let us suppose that we need to generate a given positive output voltage $V_{o}$ over a switching period. In general, it will exist a number $\bar{N}$, so that:

$$
\bar{N} \cdot V_{D C} \leq V_{o} \leq(\bar{N}+1) \cdot V_{D C} \text {, with } 0 \leq \bar{N} \leq N-1
$$

From (1) and (3), one simple way to generate the desired voltage is to divide the $N$ blocks into three categories. $\bar{N}$ blocks will be operated in "fully on" mode, each contributing with $V_{D C}$ to the output voltage. One block will be operated in PWM mode, with a duty cycle:

$$
D=\frac{V_{o}-\bar{N} \cdot V_{D C}}{V_{D C}}
$$

The remaining $N-\bar{N}+1$ blocks will be operated in "zero" mode, as described in (2). As a result:

$$
V_{o}=\bar{N} \cdot V_{D C}+D \cdot V_{D C}
$$

The same method applies in case of negative output voltage, with the only difference that the "fully on" blocks and the block doing PWM will all generate negative output voltage. It is apparent that the one described above is not the only method to generate a given output voltage. For instance, a pair of blocks operated in "zero" mode could instead be operated in "fully on" mode, generating positive and negative voltage, respectively, annihilating each other.

\subsection{Block Balancing}

The degrees of freedom in output voltage generation 
can be used to balance the load of each independent source behind the H-Bridges. From the switches topology depicted in Fig. 1, it is apparent that for a given load current $I_{L}$, the current flowing through the DC source behind the bridge is $I_{L}$ itself if the block output voltage is $+V_{D C},-I_{L}$ if the block output voltage is $-V_{D C}$; no current flows through the DC source when the block output voltage is zero. As a result, assuming $V_{o}$ and $I_{L}$ positive (reasoning is reversed in case of discordant signs of output voltage and load current), if the voltage generation method described in the previous chapter is used, in a switching period $T_{s w}, \bar{N}$ sources will deliver the same amount of energy to the load:

$$
E_{1}=T_{s w} \cdot V_{o} \cdot I_{L}
$$

The block doing PWM will deliver a smaller amount of energy to the load:

$$
E_{2}=D \cdot T_{s w} \cdot V_{o} \cdot I_{L}
$$

All the remaining blocks will deliver no energy to the load.

Whatever the technology employed for the $N$ DC sources, they will always be characterized by a certain amount of stored energy. In order to optimize the performance of the system, it is mandatory that all the sources participate evenly to the energy trade with the load. In more precise terms, the State Of Charge (SOC) of all sources should be kept balanced at all times. In order to achieve balancing, the SOC of all sources is periodically evaluated, and priorities are assigned to each block. If in the switching period to come the energy will flow from sources to load ( $V_{o}$ and $I_{L}$ have the same sign), highest priority for voltage generation is given to the block with highest SOC, while lowest priority is assigned to the most discharged source. Highest priority means that the block will be the first one to be operated in "fully on" mode, thus contributing with the maximum amount of energy given by (6). On the other hand, the block with lowest priority will be most likely operated in "zero" mode (unless output voltage close to the maximum limit is required), thus preventing further discharge.

From the reasoning above, it should be clear that the $N$ DC sources forming the basic cascaded converter need not be identical. As long as SOC can be evaluated accurately, ideal utilization of sources is still possible even in case of sources with different energy capabilities.

\section{FAULT TOLERANCE AND SELF HEALING}

In the architecture of Fig.1, all $N$ blocks of a phase leg are equivalent, and the phase output voltage is the sum of the individual blocks output voltage. That implies that if any one of the blocks is out of order, the remaining $N-1$ blocks can still operate provided that the faulty block has its output terminals short-circuited.

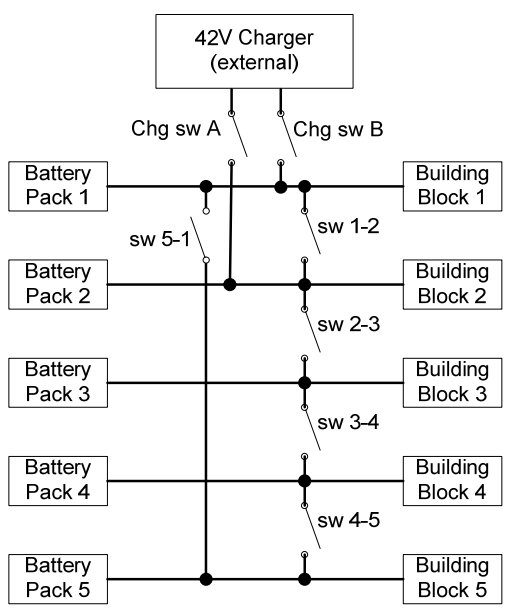

Fig. 3 - Ring connection of the battery packs for single block fault handling

Obviously, the voltage capability of the phase leg is reduced by a factor of $1 / N$, showing that if the number of levels increases, the derating following a fault becomes smaller.

As far as a block fault is concerned, self-healing can be achieved by putting a relatively inexpensive relay in parallel with the bridge output, thus bypassing the block.

It may be argued that losing a block will also reduce the driving range by the same factor as the voltage $(1 / N)$, since one battery pack is no longer used. However, if the fault is not in the battery pack itself, the energy stored in the latter can still be used by properly arranging the connections between the $N \mathrm{H}$-Bridges and the $N$ battery packs, as shown in Fig. 3 for the particular case of $N=5$. If the block $x \quad(x=1 . . N)$ is faulty, the corresponding battery pack is then connected to either block $x-1$ or $x+1$, which will therefore have a source with double capacity compared to all the others. Using the charge balancing method described in section 2.3 it is still possible to operate the system, using the whole energy available.

Again, self-healing can be achieved by performing some kind of self-test of the system that initialize the battery connections according to Table 1. Notice that

Table 1: Battery pack connection

\begin{tabular}{|l|l|l|}
\hline \multicolumn{1}{|c|}{ Operating Mode } & \multicolumn{1}{|c|}{$\begin{array}{c}\text { Charge } \\
\text { Switches }\end{array}$} & \multicolumn{1}{|c|}{ Ring switches } \\
\hline Normal operation & Both OFF & All OFF \\
\hline $\begin{array}{l}\text { Operation with } \\
\text { Block } x \text { faulty }\end{array}$ & Both OFF & $\begin{array}{l}\text { Switch } x-x-1 \\
\text { (or } x-x+1) \text { ON } \\
\text { All other switches } \\
\text { OFF }\end{array}$ \\
\hline Battery charge & $\begin{array}{l}\text { Either or } \\
\text { both ON }\end{array}$ & All ON \\
\hline $\begin{array}{l}\text { Battery charge } \\
\text { with battery pack } \\
x \text { faulty }\end{array}$ & $\begin{array}{l}\text { ON if not } \\
\text { connected } \\
\text { to } x\end{array}$ & $\begin{array}{l}\text { Switches } x-x-1 \\
\text { and } x-x+1 \text { OFF; } \\
\text { All other switches } \\
\text { ON }\end{array}$ \\
\hline
\end{tabular}




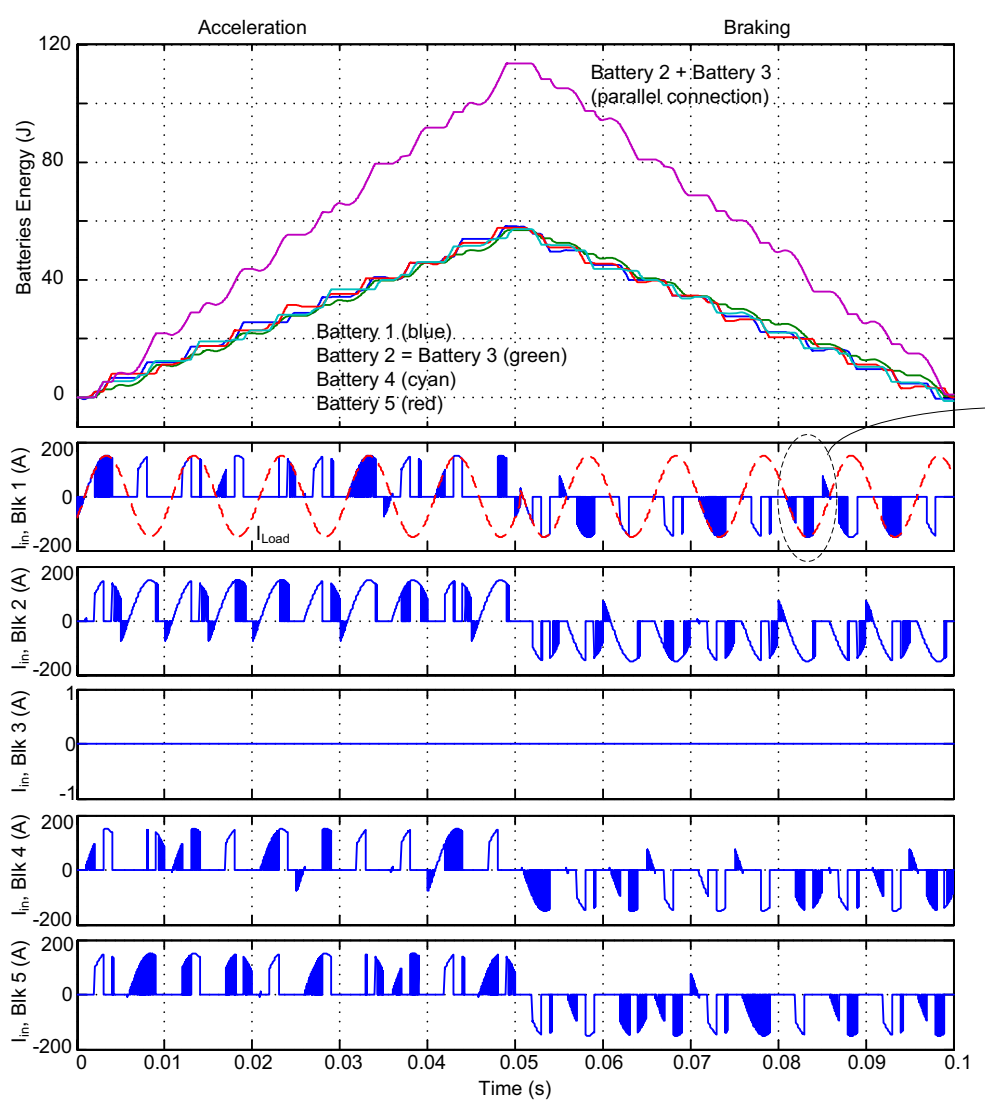

Fig. 4.a - Energy sharing during acceleration/braking with block 3 faulty and block 2 having double capacitance

the combination of switches in Fig.3 (ring topology) will also allow for parallel charging of all battery packs from a single, low voltage charger, if desired. With a redundant charge switch, it is also possible to isolate a single faulty battery pack during parallel charging, as described in Table 1 .

\section{SIMULATION RESULTS}

The cascaded multilevel converter (only one single-phase leg), operated with the voltage generation method and the charge balancing technique described in chapter 2 , has been simulated.

In the simulation of Fig. 4, the converter outputs a sinusoidal voltage having an amplitude of $90 \mathrm{~V}$, and the load current is assumed to be $150 \mathrm{~A}$ (peak); the load motor is first accelerating (p.f. $=0.85$ ) and then braking (p.f. $=-0.85)$. Throughout the whole simulation, the block 3 is assumed faulty (not operated), and block 2 is connected to two battery packs in parallel (Fig. 4.c). Results confirm that the control algorithm successfully keeps the charge on each battery pack balanced, by properly handling the switching priorities. As a consequence, average current flowing through the source behind block 2 is higher than the other blocks. However, this fact does not affect the VA ratings of the solid state switches in the H-Bridge.

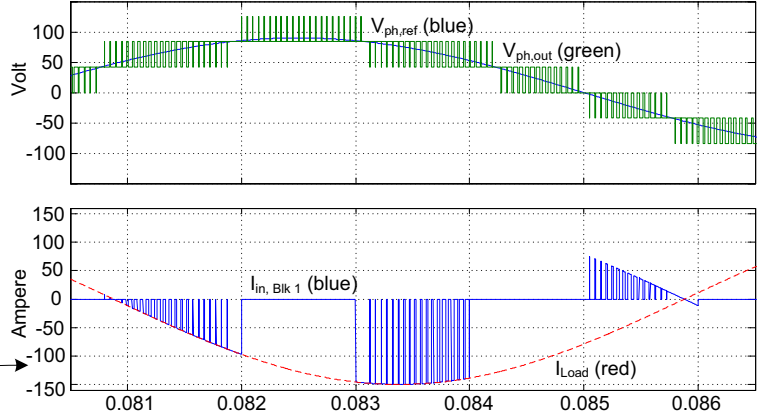

Fig. 4.b - Enlarged view of inverter output voltage (one phase) and input current to building block 1

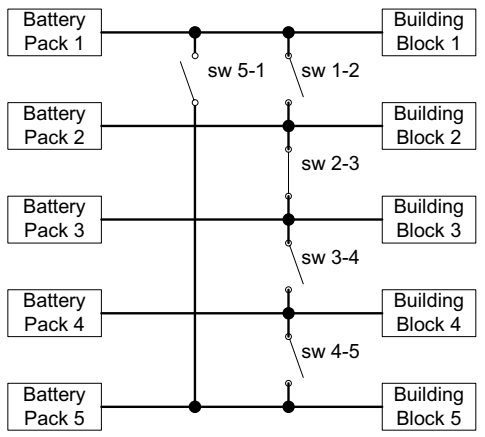

Fig. 4.c - Battery packs connection in case of Block 3 faulty

\section{EXPERIMENTAL SETUP}

The ultimate target is to build a drive system for a small city vehicle, whose electric motor characteristics are resumed in Table 2. In the final setup, wye connection of three legs based on cascaded multilevel topology (see Fig. 2) will be used. However, the basic concepts related to output voltage generation and charge balancing can be demonstrated by using a single leg topology.

\subsection{Selecting Number of Levels and Individual Voltage of Building Blocks}

In the selection of the ideal voltage ratings of the single building block, many concurrent factors have to be considered, ranging from efficiency to safety. The problem is similar to the one faced by the consortium that developed the new $42 \mathrm{~V}$ automotive standard. It seems therefore very reasonable to use that same voltage level for the building blocks. Once the block voltage is fixed, the number of levels is easily determined according to the maximum voltage requirement of the load, taking also into account some margin for better controllability and to maintain a reasonable voltage capability in case of a single block fault.

Main characteristics of the resulting converter design are resumed in Table 2. The number of building blocks 
Table 2: Final system specifications

\begin{tabular}{|c|c|}
\hline \multicolumn{2}{|c|}{ AC Motor } \\
\hline PM synchronous & $\begin{array}{l}\text { 3-phase, } 220 \mathrm{~V}, \\
3000 \mathrm{rpm}, 8 \text { poles } \\
P_{\text {rat }}=15 \mathrm{~kW}, P_{\text {peak }}=40 \mathrm{~kW}\end{array}$ \\
\hline \multicolumn{2}{|c|}{ Cascaded multilevel inverter } \\
\hline $\begin{array}{l}\text { Number of levels per } \\
\text { phase }\end{array}$ & $N=5$ \\
\hline $\begin{array}{l}\text { Voltage of each battery } \\
\text { pack }\end{array}$ & $36 V$ (nominal) \\
\hline Switching devices & $\begin{array}{l}\text { Automotive MOSFET } \\
\text { IRF3805S-7P } \\
160 \mathrm{~A}, 55 \mathrm{~V}, \\
R_{D S, \text { on }}=2.6 \mathrm{~m} \Omega @ T_{j}=25 \mathrm{C}\end{array}$ \\
\hline $\begin{array}{l}\text { Individual UC bank } \\
\text { (optional) }\end{array}$ & $\begin{array}{l}16 \times \mathrm{BCAP} 0350 \\
(2.5 \mathrm{~V}, 350 \mathrm{~F})\end{array}$ \\
\hline \multicolumn{2}{|c|}{ Hypothetical 2-level inverter } \\
\hline $\begin{array}{l}\text { Battery voltage } \\
\text { (DC-Link) }\end{array}$ & $350 \mathrm{~V}$ \\
\hline Switching devices & $\begin{array}{l}\text { Trench IGBT } \\
\text { SKM195GB066D } \\
600 \mathrm{~V}, 200 \mathrm{~A} \\
\end{array}$ \\
\hline
\end{tabular}

per phase is fixed to 5, yielding a 11 steps waveform for the phase output voltage. In the same table, a hypothetical 2-level inverter functionally equivalent to the proposed multilevel structure is reported for comparison.

\subsection{Efficiency and EMI considerations}

At first glance, it may appear that increasing the number of levels will have detrimental effect on efficiency. In fact, the number of switching devices along the load current path is always $2 N$, resulting in $2 N$ times the conduction losses (ohmic losses in case of unipolar devices like MOSFETs) of a single device. However, for technological reasons, the on-state resistance of MOSFETs of the same current ratings tends to increase more than linearly with the blocking voltage capabilities. In particular, it can be shown that for an ideal MOSFET structure, the resistance per unit area $R_{o n, s p}$ of a device is $[4,5]$ :

$$
R_{o n, s p}=K \cdot\left(B V_{p p}\right)^{2.5}
$$

where $B V_{p p}$ is the reverse voltage the device has to withstand and $K$ is a constant.

From (8) it looks clear that series connection of $2 \mathrm{~N}$ low voltage devices will result in lower conduction losses as compared to 2 devices of $N$-times higher voltage ratings. In reality, the comparison is more complex, since the optimum technology for the switching devices depends on the required voltage blocking capabilities. At $36 \mathrm{~V}$ level, as in the case of the building block of the cascaded converter proposed in this paper, MOSFETs are the correct choice; however,

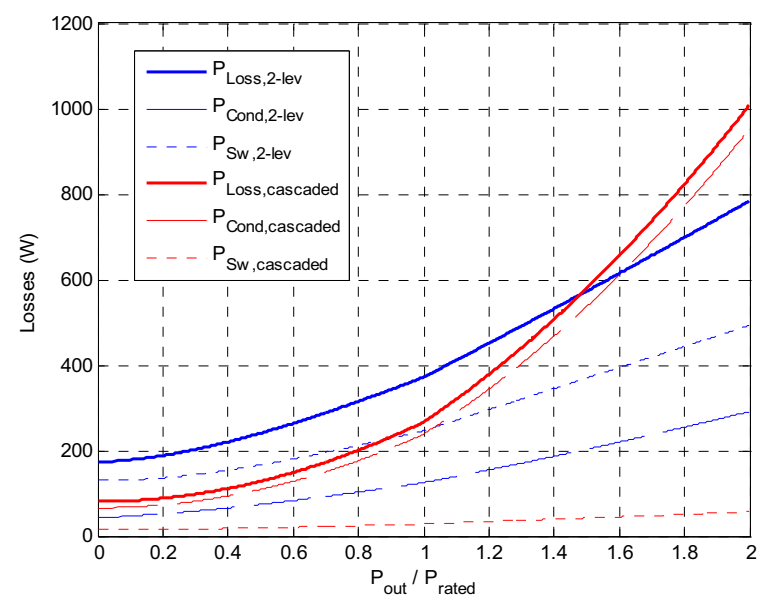

Fig. 5 - Comparison of losses in the switching devices

an hypothetic 2-level inverter driving the same motor load would have a DC-link voltage higher than $300 \mathrm{~V}$, making IGBT the preferred device, as shown in Table 2.

Fig. 5 shows a comparison between losses in the proposed cascaded converter and in a standard 2-level inverter of equivalent ratings; the comparison is performed at rated inverter output voltage, varying the power absorbed by the load.

Losses in the IGBT inverter are calculated using the methods first presented in [6]; similar results can also be obtained using the loss calculator developed by the device manufacturer [7]. In the case study, switching frequency of the IGBT inverter is fixed to $12 \mathrm{kHz}$.

Conduction losses in the MOSFET-based cascaded inverter are assumed to be purely resistive, since the anti-parallel diodes (see Fig. 1) will only conduct current for a very short period of time (the dead time of the block being operated in PWM mode):

$$
P_{\text {cond,MOSFET }}=R_{D S} \cdot I_{D}^{2}
$$

In evaluating (9), $R_{D S}$ should be the resistance of the device at the target junction temperature, here assumed to be $100^{\circ} \mathrm{C} ; I_{D}$ is the rms value of the load current.

For simplicity, PWM switching frequency of the cascaded inverter is also fixed to $12 \mathrm{kHz}$. This will actually give a much better voltage waveform than the 2-level inverter (cfr. Fig. 4b). However, since only one out of $N$ blocks is operated in PWM mode in each switching period, the equivalent switching frequency will be $N$ times lower. This, added to the fact that MOSFET devices exhibit in general much better switching characteristics than IGBT, due to the unipolar nature of the current inside the semiconductor [5], explains why switching losses are so small in the case of cascaded inverter.

From Fig. 5 it results that the IGBT inverter has lower losses when operated at peak power, but the cascaded inverter is more efficient all the way from no load up to about 1.5 times the rated power. Since a typical driving cycle will imply operation below this breakeven point for most of the time, it is reasonable to 
conclude that the cascaded inverter has potential for better efficiency, overall.

Moreover, since losses in the cascaded inverter are dominated by conduction losses, it is possible in principle to modify the design process for the switching devices, yielding a MOSFET with lower conduction losses at the expense of higher switching losses; in this particular application, this would result in an overall reduction of losses, making the proposed architecture even more favorable.

Electromagnetic emissions from the converter are mainly due to the steep current slopes resulting from PWM. There are at least two reasons why the cascaded multilevel topology described so far is expected to be superior in terms of EMI when compared to standard hard-switched topologies. First of all, due to the multilevel structure, voltage steps due to the switching devices are reduced in height to the individual DC link voltage. Secondly, and perhaps even more important, since the switching losses of the converter are shown to be very low, it is possible to slow down the commutation of each MOSFET, reducing the current slope and therefore EMI considerably; the resulting increase in switching losses due to the slower commutation will not affect the efficiency in any significant way.

Slowing down the switching speed of MOSFETs from their rated capabilities will also have another very favorable consequence: reduction of the over-voltage peak across the switch at each commutation. This allows for the use of lower voltage devices, like the one mentioned in Table 2, having $55 \mathrm{~V}$ reverse blocking capabilities when the DC link voltage can be as high as $42 \mathrm{~V}$. Such a small voltage margin would hardly be feasible otherwise, even if the breakdown capabilities of modern MOSFETs are exploited. As already mentioned, the use of devices with lower blocking voltage will reduce the conduction (dominating) losses.

\subsection{System Layout and Control Structure}

As suggested by Figg.1-2, from the system integrator point of view the system is made up of identical modules (the Building Blocks), embedding DC source, $\mathrm{H}$-Bridge, relays and digital control. The two output terminals are connected to form the desired topology (could be single leg, wye, delta, etc.). Extra connections are needed for the battery charger and to achieve redundancy as described in chapter 3 . However, if the building block is properly engineered, the connection can be considerably simplified, as suggested in Fig. 6 . Moreover, each converter leg of a Wye topology can be in physically different locations on board of the vehicle, and the interconnection between them is limited to one power line. In addition, each leg has to be connected to one motor phase, to the charger and to the master controller.

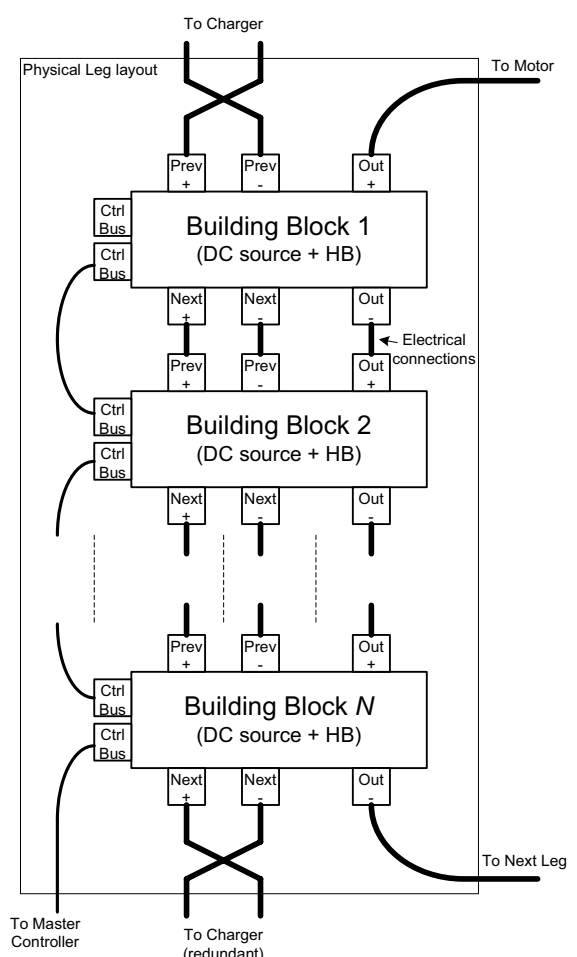

Fig. 6 - Leg physical layout and suggested cabling

Such a decentralized converter will obviously need a dedicated control structure. In this work, a standard motor controller operates as master and calculates the voltage needed at the load terminals. A second control module, also located at master level, will then figure out a proper way to synthesize the required voltage, with the additional control target of keeping the SOC of all blocks properly balanced. The building blocks are slave modules; they communicate to the master their voltage availability and their current SOC, and receive in turn the switching command.

In order to simplify cabling and to increase reliability, the communication within the system is purely serial. A very strict time triggered protocol has been implemented to properly synchronize the switching of all blocks, and much effort has been devoted to give the communication system the necessary degree of reliability. Due to the limited space, this aspect will not be further discussed in this paper.

\subsection{First Operational Prototype}

In order to validate the proposed concepts, and to start working towards the final system, a first fully operational prototype has been built. The system consists of a single leg converter made of two identical building blocks, having the characteristics of the final ones, except that the UC part is not present. The local DC link of each building block is made up of a series connection of three standard $12 \mathrm{~V}, 10 \mathrm{Ah}$ sealed lead acid batteries, resulting in $36 \mathrm{~V}$ rated voltage. Solid state devices used for the H-Bridges are the ones in Table 2. 

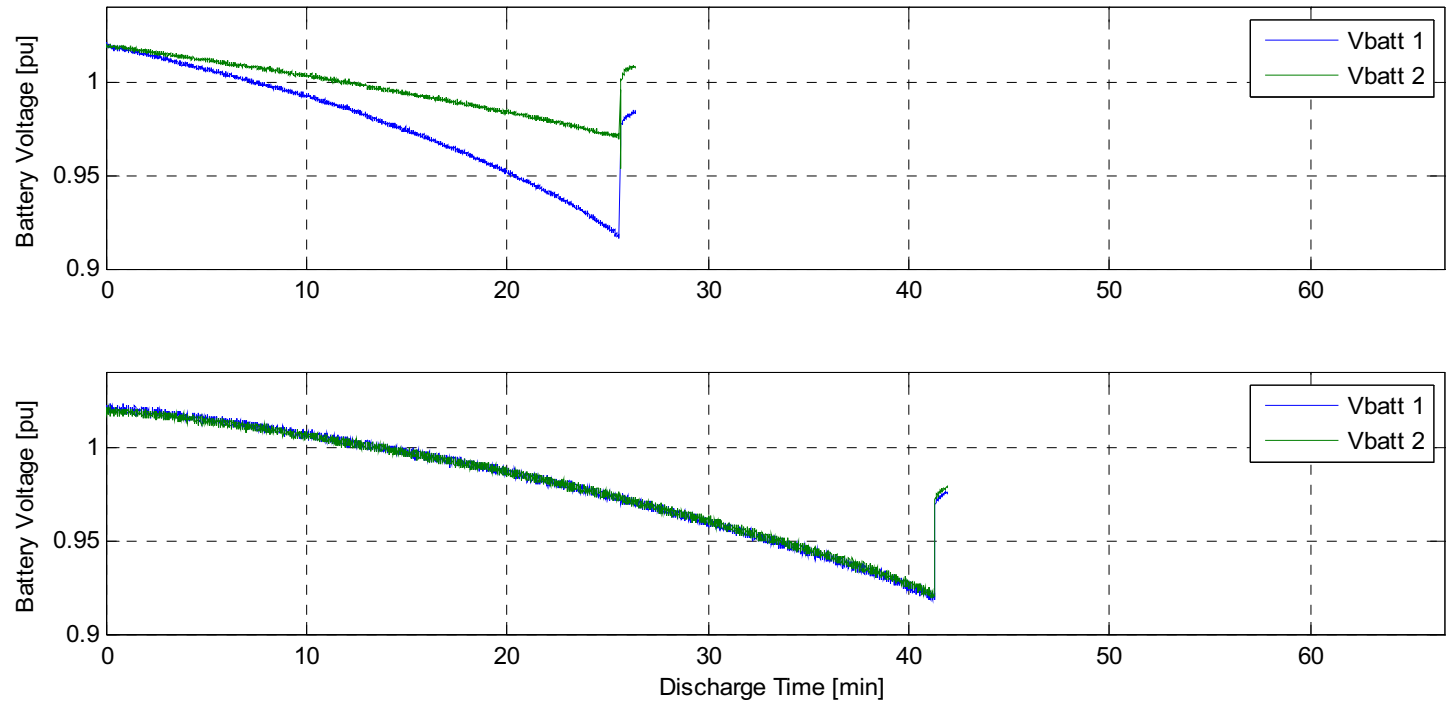

Fig. 7 - Experimental results of battery discharge at constant load voltage $(0.7 \mathrm{pu})$ and constant load current $(0.7 \mathrm{pu})$; From top to bottom:

(a) - With no control for energy sharing; block1 has always higher priority than block 2;

(b) Energy sharing by dynamic assignment of priorities based on on-line monitoring of SOC;

The control hardware features all the characteristics of the final setup, and so does the time-triggered serial protocol, making expansion of the system by either adding more blocks in series or forming a Wye connection to achieve a three phase output rather straightforward.

\subsection{Experimental Results}

The basic experiment of Fig. 7.a shows a constant current discharge of the 5 level system $(N=2)$ with no charge balancing. Output voltage of the converter is fixed to $50.4 \mathrm{~V}(0.7 \mathrm{pu}$, having assumed the base voltage equal to the nominal voltage of the batteries), and the load is a DC motor, absorbing about $10 \mathrm{~A}$ throughout the experiment. Discharge is stopped as soon as the lowest battery voltage reaches $0.92 \mathrm{pu}$ (about $33 \mathrm{~V}$ ). End of discharge is easily noticed in the figure, since the terminal voltage of the batteries suddenly rises to the open-circuit value when the current drops to zero. Priorities in the modulation algorithm are fixed and block 1 has always higher priority than block 2 . As expected, the battery pack connected to block 1 run out of energy faster than block 2, resulting in about $26 \mathrm{~min}$ of available operating time before the minimum allowed terminal voltage is reached.

In Fig. 7.b, all conditions above remain unchanged, but the proposed charge balancing algorithm is enabled and switching priorities between blocks are dynamically assigned by the master controller. It can be seen that both blocks discharge equally, resulting in optimal utilization of the energy, as witnessed by the extended operating time of about $41 \mathrm{~min}$.

It should be noticed that during this experiment the SOC of each block is evaluated locally by the corresponding slave with a very rough algorithm, assuming the SOC to be a function of just the battery voltage and the current. This is obviously not the best evaluation method and should certainly be improved in the future; however, the master controller responsible for global charge balancing will not be affected by the change, since it only receives information about SOC of each block, and need not to know how that number is evaluated.

In the experiment of Fig. 8, one extra battery pack $(36 \mathrm{~V}, 10 \mathrm{Ah})$ has been connected in parallel to block 2 . This condition resembles the case of a system with $N=3$, with one of the three blocks being out of order and its battery pack connected instead to one of the healthy blocks in order to be able to use all the energy available in the system, as described in chapter 3. Results confirm that the three packs discharge equally up to a point very close to the end of discharge. The resulting operating time is about $61 \mathrm{~min}$ that is about 1.5 times the operating time obtained in the experiment of Fig. 7.b, where the available energy was 1.5 times smaller. Non ideal balancing towards the end of the test is due to the poor evaluation of the SOC of each block, as pointed out earlier, and also to the poorly matched characteristics of the batteries in use. Fig. 8.b shows the average current flowing on each of the three battery packs during the test $(1.0 \mathrm{pu}$ is equal to $10 \mathrm{~A}$, which is the $1 \mathrm{C}$ ratings of the batteries in use). Ideally, they should be identical throughout the experiment. While the difference between $I_{1}$ and the other currents is mostly due to poor SOC estimation, the difference between $I_{2}$ and $I_{3}$ is fully due to unbalanced battery packs, since the terminals of the two packs are connected in parallel with no control whatsoever for current sharing. In particular, it may be noticed that the sharing algorithm is trying to assign lower priority to the most discharged block 1 towards the end of the experiment, but even so, the voltage across that block 

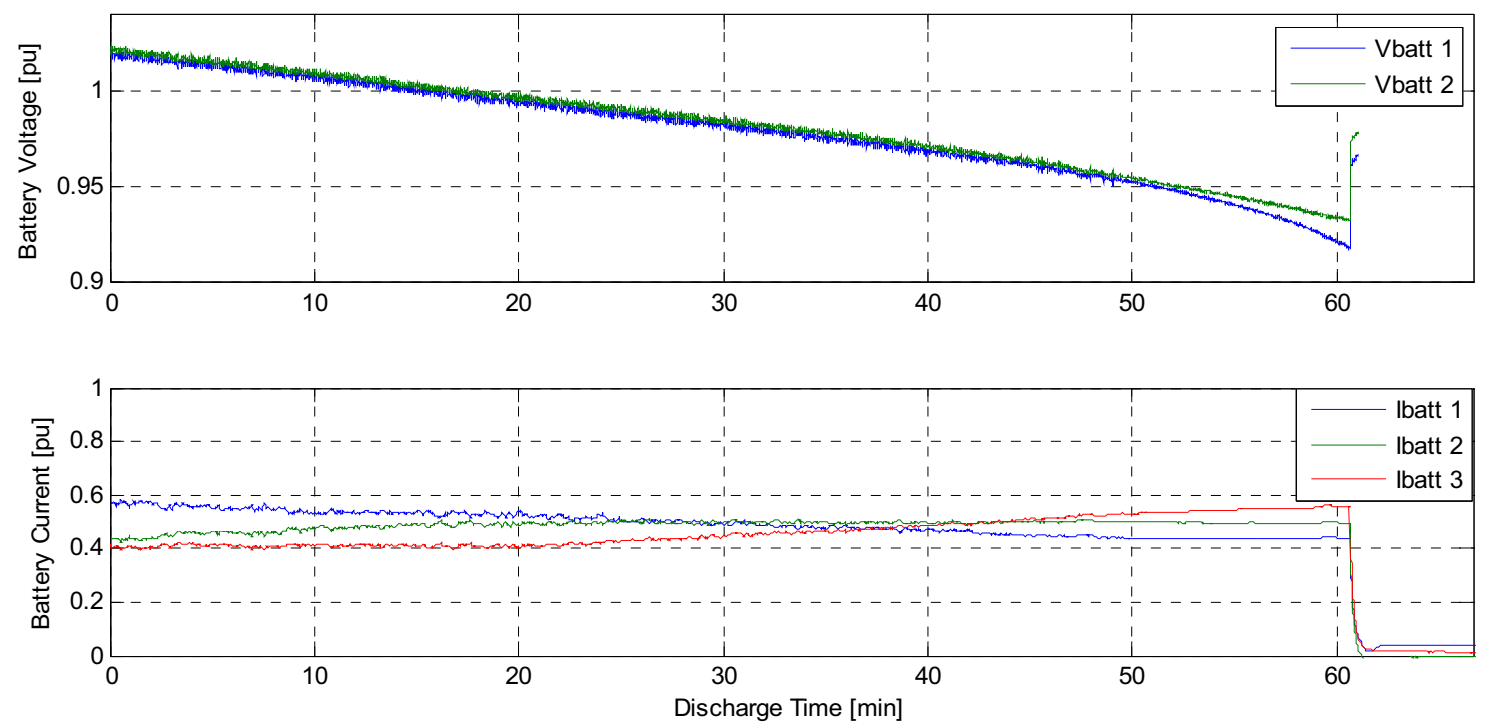

Fig. 8 - Experimental results of constant power discharge of a system composed by two H-Bridges and three battery packs, two of which are connected in parallel; From top to bottom:

(a) - Terminal voltage at the input of each H-Bridge (same as Fig. 5.c);

(b) Average Current flowing from each battery pack (packs 2 and 3 are in parallel);

falls more quickly than the voltage across the block who is given higher priority (and who is asked for more current).

Even with all limitations and inaccuracies discussed above, operating range close to ideal has been achieved.

\section{CONCLUSION}

Cascaded multilevel topology is a promising alternative to be used as interface between the energy storage devices (batteries and/or UCs) and the electrical load on board of electric vehicles. Besides the advantages in terms of voltage quality, safety, EMI and, most likely, efficiency, the cascaded inverter has the unique feature of being inherently redundant, making it an ideal starting point to built highly dependable systems for applications with very strict requirements in terms of reliability.

Several aspects related to both operational principle and practical realization of a cascaded multilevel converter suitable for EVs has been analyzed, with special emphasis on self-healing and post-fault operation.

A first working prototype has been built, and the results obtained so far are promising; it is believed that the proposed topology, if properly equipped with a control system designed for reliability and fault-tolerance, can be applied in special occasions when quality and safety concerns offset the increase of complexity that the multilevel, multicell topology brings with it.

\section{REFERENCES}

[1] L.M. Tolbert, F.Z. Peng, "Multilevel converters for large electric drives," IEEE Applied Power Electronics
Conference, 1998, pp. 530-536.

[2] S. Pay, Y. Baghzouz, "Effectiveness of Battery-Supercapacitor combination in Electric Vehicles," IEEE Bologna PowerTech Conference, 2003.

[3] P. Barrade, A. Rufer, "Supercapacitors as energy buffers: a solution for elevators and for electric busses supply," Power Conversion Conference, 2002, pp. 1160-1165.

[4] N.Mohan, T.M. Undeland, W.P. Robbins "Power Electronics", John Wileys \& Sons, 3rd edition, 2003.

[5] B.J. Baliga, "Power Semiconductor Devices", PWS Publishing Co., 3rd edition, 1996.

[6] J. Kolar, H. Ertl, F.C. Zach, "Influence of modulation methods on PWM converter losses", IEEE Trans. On Ind. Applicat., Vol. 27, No. 6, 1991, pp. 1063-1075.

[7] http://semisel.semikron.com

\section{BIOGRAPHIES}

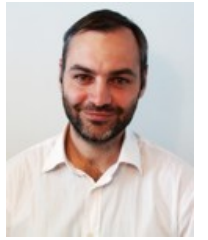

Giuseppe Guidi graduated from the University of L'Aquila, Italy, in 1995, and was cooperative researcher at The University of Tokyo, Japan in 1996. From 1998 to 2001 he was with Fuji Electric R\&D Ltd, Tokyo, Japan, as R\&D engineer; he then joined SIEI Spa, Gerenzano, Italy, where he was senior drive engineer until 2004, when he joined the Department of Electrical Power Engineering at NTNU, Norway, where he is currently working towards his $\mathrm{PhD}$.

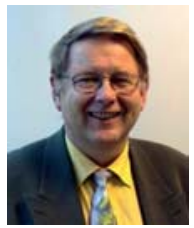

Tore M. Undeland has been Professor since 1984 at NTNU, Trondheim, Norway, and is also Adjunct professor at Chalmers, Sweden. IEEE Power Electronics Society board member in 6 years, and Past president of EPE - European Power Electronics Association. His research interests are: Design of converters and Conversion and control in Renewable Energy. He has published about 100 papers, and is coauthor of the much used textbook: Mohan, Undeland, Robbins: Power Electronics, John Wiley \& Sons, 3rd Ed 2003 